\title{
Temporal disparity in the induction of matrix metalloproteinases and tissue inhibitors of metalloproteinases after thoracic aortic aneurysm formation
}

\author{
John R. Barbour, MD, ${ }^{\text {a }}$ Robert E. Stroud, MS, ${ }^{a}$ Abigail S. Lowry, BS, ${ }^{a}$ Leslie L. Clark, MS, ${ }^{a}$ Allyson M. Leone, BS, \\ Jeffery A. Jones, PhD, ${ }^{a}$ Francis G. Spinale, MD, PhD, ${ }^{a, b}$ and John S. Ikonomidis, MD, $\mathrm{PhD}^{\mathrm{a}}$
}

From Cardiothoracic Surgical Research, Division of Cardiothoracic Surgery, Medical University of South Carolina, ${ }^{\mathrm{a}}$ Charleston, $\mathrm{SC}$, and the Ralph H. Johnson Veterans Affairs Medical Center, ${ }^{\mathrm{b}}$ Charleston, SC.

Supported by National Institutes of Health/ National Heart, Lung, and Blood Institute R01 grants HL075488-01 and HL059165-07 and the Research Institute of the Department of Veterans Affairs Award.

Received for publication Jan 29, 2006; revisions received May 11, 2006; accepted for publication May 22, 2006.

Address for reprints: John S. Ikonomidis, MD, PhD, Division of Cardiothoracic Surgery, Medical University of South Carolina, Suite 409 CSB, 96 Jonathan Lucas St, Charleston, SC 29425 (E-mail: ikonomij@ musc.edu).

J Thorac Cardiovasc Surg 2006;132:788-95 $0022-5223 / \$ 32.00$

Copyright (๑) 2006 by The American Association for Thoracic Surgery

doi:10.1016/j.jtcvs.2006.05.052
Background: An important component of matrix remodeling during thoracic aortic aneurysm progression is the balance between matrix metalloproteinases and their endogenous inhibitors (tissue inhibitors of metalloproteinases). However, whether and to what degree matrix metalloproteinase/tissue inhibitor of metalloproteinases profiles change over time with an evolving thoracic aortic aneurysm remains unclear.

Methods: Descending thoracic aortic aneurysms were induced in mice (FVB strain, 15 minutes of $0.5 \mathrm{~mol} / \mathrm{L} \mathrm{CaCl}{ }_{2}$ exposure) and followed for 24 hours, 72 hours, 1 week, 2 weeks, 4 weeks, or 8 weeks (each group, $n=13$ ). Thoracic aortic aneurysm size was determined by means of video micrometry, and immunoblotting was used to measure aortic matrix metalloproteinase 2, 8, 9, and 12 and tissue inhibitor of metalloproteinases 1 and 4 levels (expressed as a percentage of control values, $n=13$ ).

Results: Increased aortic diameter was detected by 72 hours and reached a maximal size at 4 weeks $(135 \% \pm 4 \%$ increase from baseline, $P<.05)$, which is consistent with thoracic aortic aneurysm progression. Active matrix metalloproteinase 8 (collagenase) levels increased at 72 hours $(178 \% \pm 49 \%, P<.05$ from control), and active matrix metalloproteinase 12 (elastase) levels increased by 24 hours $(138 \% \pm$ $11 \%, P<.05)$, whereas active matrix metalloproteinase 2 levels increased at 72 hours and 1 week after thoracic aortic aneurysm induction (72 hours: 158\% \pm $12 \%, 1$ week: $162 \% \pm 19 \% ; P<.05)$. At 1 week after thoracic aortic aneurysm induction, active matrix metalloproteinase 9 and 12 levels decrease (matrix metalloproteinase 9: $55 \% \pm 5 \%$; matrix metalloproteinase 12: $63 \% \pm 5 \% ; P<.05$ ); however, matrix metalloproteinase 9 and 12 levels were increased from these values at 4 and 8 weeks $(P<.05)$. Tissue inhibitor of metalloproteinases 1 levels were decreased at 1 week $(52 \% \pm 15 \%, P<.05)$ and later returned to control values, whereas tissue inhibitor of metalloproteinases 4 levels increased at the late thoracic aortic aneurysm time points (4 weeks: $278 \% \pm 46 \%$; 8 weeks: $213 \% \pm 40 \% ; P<.05$ ).

Conclusions: These findings show 2 phases of matrix metalloproteinase abundance during murine thoracic aortic aneurysm formation. The late tissue inhibitor of metalloproteinases 4 increase might explain prevention of further aortic dilation past 4 weeks. Unique matrix metalloproteinase/tissue inhibitor of metalloproteinases temporal relationships occurred during the natural history of thoracic aortic aneurysm progression that might hold both diagnostic and therapeutic relevance.

$\mathrm{T}$ horacic aortic aneurysms (TAAs) represent a serious and potentially lethal disease with high mortality and morbidity rates. TAA formation and progression is a multifactorial process that involves both cellular and extracellular processes. ${ }^{1,2}$ Ascending and descending TAAs likely differ in cause and natural progression, ${ }^{3}$ and anatomic and cellular differences between the ascending 


\section{Abbreviations and Acronyms \\ $\mathrm{ECM}=$ extracellular matrix \\ $\mathrm{MMP}=$ matrix metalloproteinase \\ $\mathrm{TAA}=$ thoracic aortic aneurysm \\ TIMP $=$ tissue inhibitor of metalloproteinases}

and descending aorta might explain why TAAs that develop in these areas differ. With respect to the extracellular matrix $(\mathrm{ECM})$, degradation and remodeling universally occurs with descending TAAs, but the underlying mechanisms remain poorly understood.

ECM degradation in aneurysm tissue is caused in part by a family of endopeptidases termed matrix metalloproteinases (MMPs). ${ }^{4,5}$ Several members of the MMP family have been implicated in the pathogenesis of aortic aneurysms, including gelatinase A (MMP-2), gelatinase B (MMP-9), and macrophage elastase (MMP-12). ${ }^{6-9}$ The major structural component that lends strength to the aortic wall is elastin. Elastin fiber fragmentation is a frequent histologic finding in aneurysmal tissue. ${ }^{10}$ MMP-12, released from macrophages, has the ability to directly degrade elastin within the aortic wall, facilitating aneurysmal dilatation with the loss of structural integrity. Neutrophil collagenase (MMP-8) and MMP-9 are released in their preformed states from neutrophils in the early stages of inflammation. In addition, it has been established that the endogenous tissue inhibitors of the MMPs (TIMPs) play a role in the modulation of MMP activity. ${ }^{11,12}$ Radiolabeled MMP inhibitors, combined with in vivo scintigrapghy in experimental animal models, have opened the door for noninvasive clinical diagnostic methods in MMP-related diseases. ${ }^{13}$ Although previous studies have demonstrated clear roles for these specific MMPs and TIMPs in aneurysmal disease, no study to date has determined the relative changes in abundance over the course of aneurysmal development. We hypothesized that in a model of descending TAA,${ }^{14}$ temporal changes in these critical MMP and TIMP types occur throughout TAA progression. Hence the present study examines the changes in these species after experimental descending TAA induction.

\section{Methods \\ Experimental Design}

Animals used in the study were adult wild-type FVB mice $(\mathrm{n}=$ 91). Equal numbers of male $(\mathrm{n}=47,52 \%)$ and female $(\mathrm{n}=44$, $48 \% ; P=.54$ ) mice were used, and all mice were between 8 and 12 weeks of age at the time of the initial operation. Mice were randomly assigned to one of 6 groups for terminal harvest and analysis 24 hours $(\mathrm{n}=13), 72$ hours $(\mathrm{n}=13), 1$ week $(\mathrm{n}=13)$, 2 weeks $(n=13), 4$ weeks $(n=13)$, or 8 weeks $(n=13)$ after the initial operation. From each group, the aortas from 3 mice were formaldehyde fixed for histologic studies, and the aortas from 10 mice were frozen for biochemical analysis. One mouse each from the 24-hour and 1-week groups were excluded because of inadequate tissue. For biochemical analysis, the above animals were compared with age-matched, control unoperated animals $(\mathrm{n}=10)$. All animals were treated and cared for in accordance with the National Institutes of Health "Guide for the Care and Use of Laboratory Animals" (National Research Council, Washington, DC, 1996), and the protocols were approved by the Medical University of South Carolina Institutional Animal Care and Use Committee (protocol no. 2146).

\section{Operative Procedure}

Murine TAA production was induced as previously described. ${ }^{12,15}$ Briefly, periadventitial calcium chloride $\left(\mathrm{CaCl}_{2}\right)$, which has been shown to lead to structural disruption of the medial layer of the aortic wall and the induction of a localized inflammatory response, was applied to the abluminal surface of the descending thoracic aorta to induce aneurysmal dilatation. ${ }^{14}$ This experimental model of aortic aneurysm was selected for this study, as opposed to the elastase perfusion model, because the modest dilation it produces likely mimics the early stages of aneurysmal formation.

Anesthetized and orotracheally intubated animals were subjected to fifth intercostal space thoracotomy with exposure of the descending thoracic aorta. Aortic diameter measurements were obtained. A sponge soaked in $0.5 \mathrm{~mol} / \mathrm{L} \mathrm{CaCl}_{2}$ was placed on the distal half of the descending thoracic aorta for 15 minutes. After 15 minutes, the sponge was removed, the chest was irrigated, and the lung was re-expanded. The operative mortality rate was $20 \%$. The mice then survived for the time points indicated before terminal study and aortic harvest.

\section{Aortic Diameter Measurements}

Aortic diameter, as assessed by means of video micrometry, was measured for each mouse at both the baseline and predetermined time point terminal operation. Digital images of the descending thoracic aorta were obtained by using a color CCD camera (KP DZ0B, Hitachi Kokusai Electric Inc, Tokyo, Japan) linked to a laptop computer with digital imaging software (WinTV2000, Hauppauge Computer Works, Inc, Hauppage, NY). Aortic diameter measurements were made through a digital video caliper (DMZR, Techni-Quip, Polk City, Fla). Terminal aortic size was expressed as a percentage increase from respective baseline measurement.

\section{Aortic Harvest}

With the animal anesthetized, the left thoracotomy was reopened and extended beneath the xiphoid process. Terminal aortic diameter measurements were obtained in vivo before death to ensure that the aorta was sufficiently pressurized. The aorta was then carefully harvested from its root to the aortic bifurcation. The descending thoracic portion was then divided from the ascending aorta, as well as the abdominal aorta.

\section{MMP and TIMP Abundance}

For this study, the relative abundances of MMPs/TIMPs were examined by using quantitative immunoblotting techniques, which have been described in detail previously. ${ }^{16}$ Briefly, $10 \mu \mathrm{g}$ of aorta extract was loaded onto a $4 \%$ to $12 \%$ Bis-Tris gradient gel and subjected to fractionation by means of electrophoresis. The fractionated proteins 
were transferred to nitrocellulose membranes $(0.45 \mu \mathrm{m}$, Bio-Rad $)$ and incubated in antiserum $(0.4 \mu \mathrm{g} / \mathrm{mL}$ in $5 \%$ nonfat dry milk/phosphatebuffered saline) specifically corresponding to MMP-8 (PC493, Oncogene), MMP-2 (AB19015, Chemicon, Temecula, Calif), MMP-9 (AB804, Chemicon), and MMP-12 (AB19051, Chemicon). Because MMPs exist in both a latent and active form, the molecular weight bands corresponding to each were quantified. Positive controls (recombinant enzyme) were included in the immunoblotting approach. The TIMPs were measured in identical fashion by using specific antisera for TIMP-1 (AB770, Chemicon) and TIMP-4 (AB816, Chemicon). A secondary peroxidase-conjugated antibody was then applied (1:5000, 5\% nonfat dry milk/phosphate-buffered saline), and signals were detected with a chemiluminescent substrate (Western Lightning Chemiluminescence Reagent Plus, Perkin Elmer, Norwalk, Conn). The film was developed in a Konica SRX-101A medical film processor and analyzed with Gel-Pro Analyzer software (version 3.1.14, Media Cybernetics Inc, Silver Spring, Md). ${ }^{17}$

\section{Statistical Analysis}

All statistical procedures were performed with the Stata statistical software package (Stata Corp, College Station, Tex). Terminal aortic diameter measurements were analyzed by using the Wilcoxon matched-pairs test to determine differences from baseline values. Comparisons of aortic diameter between groups were made with Mann-Whitney $U$ tests to determine statistical differences. MMP immunoblotting data were analyzed by using KruskalWallis analysis of ranks and then subjected to Mann-Whitney $U$ tests to determine differences between groups. Data are presented as means \pm standard error of the means.

\section{Results}

Aortic diameter increased by 72 hours, which is consistent with TAA formation and reached a maximal size at 4 weeks after $\mathrm{CaCl}_{2}$ exposure (Figure 1).

Levels of the latent form of MMP-8 $(85 \mathrm{kd})$ decreased from control levels at 72 hours $(78 \% \pm 11 \%, P=.06)$ and reached lowest relative abundance at 1 week after TAA induction operations $(44 \% \pm 6 \%, P<.05)$. In contrast, active MMP-8 (58 kd) levels were increased at 72 hours (Figure 2, A) and returned to control values by 1 week.

Latent MMP-12 (54 kd) levels were increased from control levels at 72 hours $(115 \% \pm 6 \%, P<.05)$ after TAA induction. Latent MMP-12 levels were decreased from control levels, however, at 1 week $(81 \% \pm 5 \%, P<.05)$ and returned to control levels at 2 weeks and beyond. Active MMP-12 (45 kd) levels were increased at 24 hours and decreased at 1 week before returning to control levels at 8 weeks (Figure 2, B).

Latent MMP-2 (72 kd) levels demonstrated no change at any time point in this study. In contrast, active MMP-2 $(62 \mathrm{kd})$ levels increased significantly at 72 hours and 1 week after TAA induction operations (Figure 3,A) and then returned to control values at 2 weeks and beyond. Latent MMP-9 (92 kd) levels reached their greatest abundance at 72 hours after TAA operations $(268 \% \pm 49 \%, P<.05)$ and decreased from control levels at 2 weeks $(79 \% \pm 7 \%, P<$

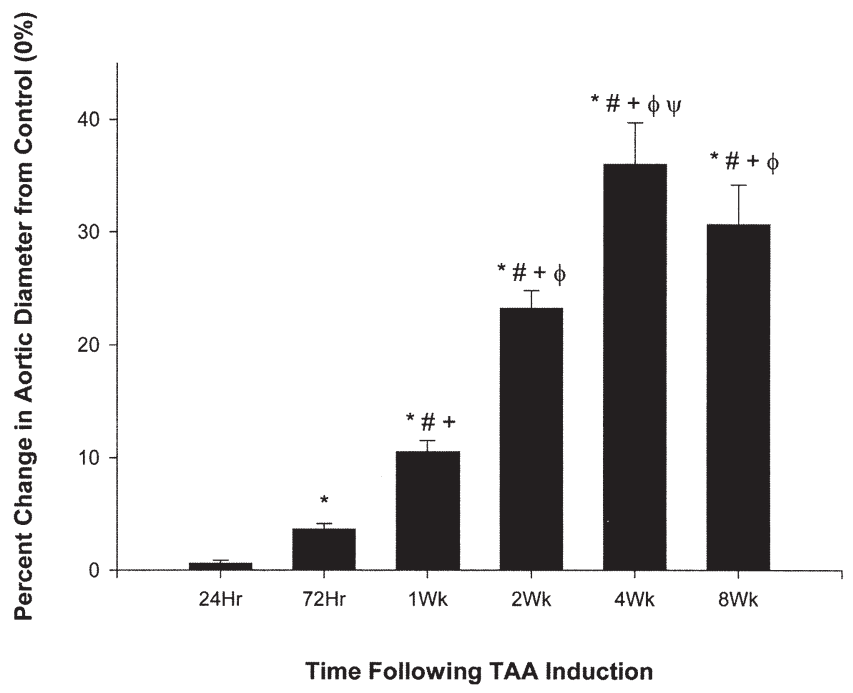

Figure 1. Change in aortic diameter between baseline and terminal values, as measured by means of video micrometry. There was no change in aortic size at $\mathbf{2 4}$ hours; however, all time points 72 hours and beyond demonstrated statistical increase in size from baseline. Aortic size reached its greatest value at 4 weeks after thoracic aortic aneurysm (TAA) operations. ${ }^{*} P<.05$ from control, \#P<.05 from 24 hours, $+\boldsymbol{P}<.05$ from 72 hours, $\phi P<.05$ from 1 week, $\Psi P<.05$ from 2 weeks.

$.05)$. Active MMP-9 (88 kd) levels were significantly decreased at 1 week and increased to control levels at 2 weeks (Figure 3, B). At 8 weeks, active MMP-9 levels were maximally increased compared with levels seen at the other time points in this study.

TIMP-1 levels decreased from control levels at 1 week (Figure 4, A) and then returned to control levels thereafter. TIMP-4 levels were not changed at the early time points in this study; notably, however, at 4 weeks, TIMP-4 levels were increased to almost $300 \%$ of unoperated control values and remained increased at 8 weeks (Figure $4, B$ ).

\section{Discussion}

Aortic aneurysmal disease is characterized by structural alterations of the aortic wall resulting from the degradation of ECM components. These changes are associated with an inflammatory infiltrate and altered production of MMPs, which mediate the degradation of ECM proteins. ${ }^{5,8,9}$ This study was designed to follow the active forms of major MMP types from representative classes of proteases implicated in TAA formation (MMP-2, MMP-8, MMP-9, and MMP-12), as well as their endogenous tissue inhibitors (TIMP-1 and TIMP-4), throughout the course of TAA progression in a reproducible murine model. These results demonstrated a distinctive biphasic profile of MMP and TIMP abundance over the 8-week study period. Specifi- 
MMP-8

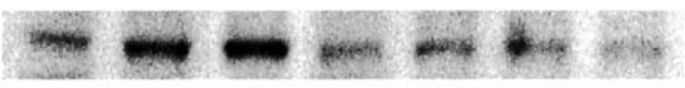

$\leftarrow 58 \mathrm{kDa}$

A.

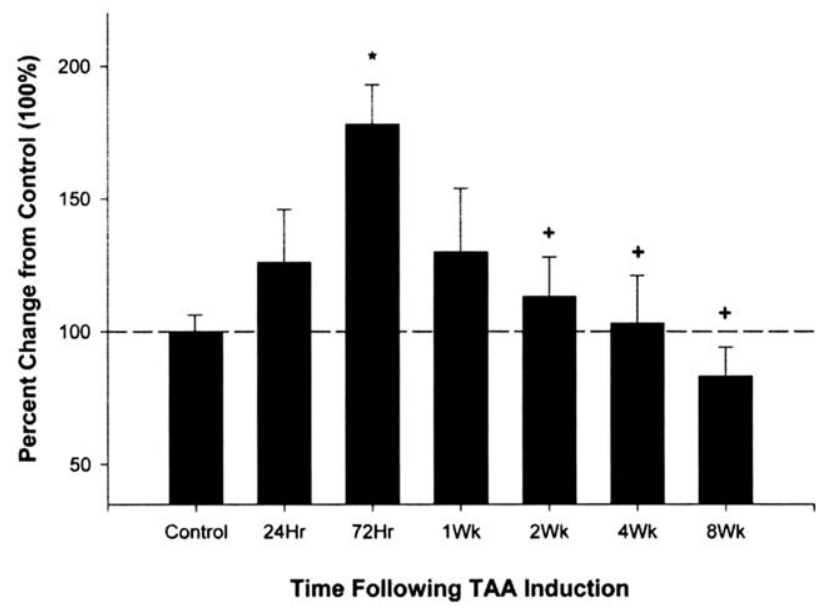

Figure 2. A, The representative immunoblot demonstrates the 58-kd band characteristic of active matrix metalloproteinase (MMP) 8. MMP-8 showed early increased abundance, which peaked at 72 hours after the operation; however, MMP-8 levels were found to be near control values by 1 week and remained near control values at all other time points in this study. ${ }^{*} \boldsymbol{P}<.05$ from control, $+\boldsymbol{P}<$ .05 from 72 hours. $B$, The representative immunoblot demonstrates the $45-k d$ band characteristic of active MMP-12. MMP-12 levels were increased early and increased from control values at 24 hours. After this initial spike, MMP-12 levels were decreased in abundance at 1 week and returned to normal at 2 weeks. ${ }^{*} \boldsymbol{P}<.05$ from control, $\# \boldsymbol{P}<.05$ from 24 hours, $+P<.05$ from 72 hours, $\phi P<.05$ from 1 week, $\Psi P<.05$ from 2 weeks. TAA, Thoracic aortic aneurysm.

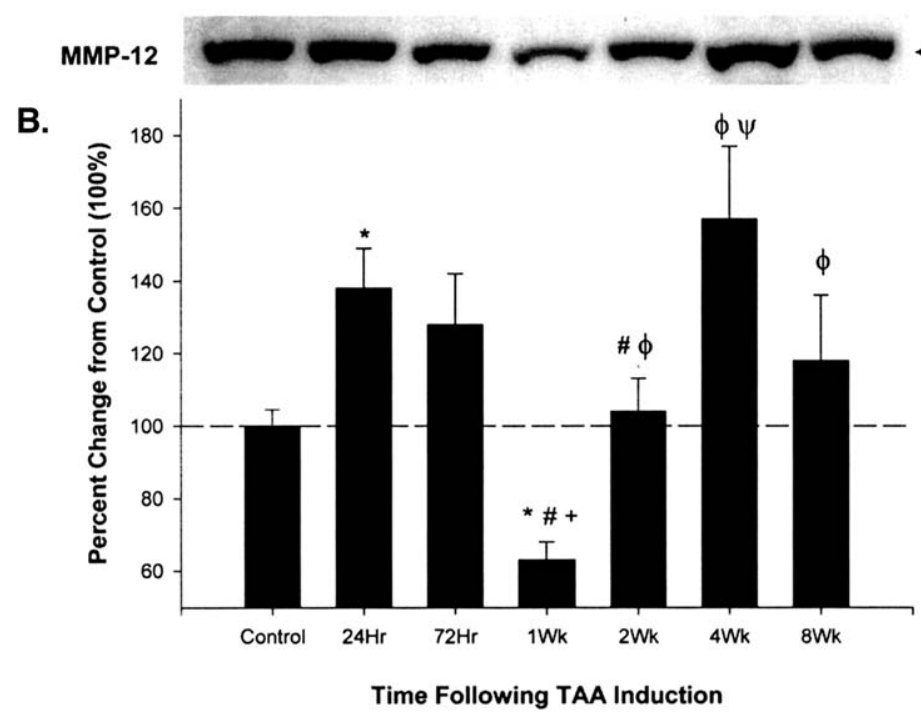

cally, this model demonstrated an early phase of aneurysmal expansion characterized by relative increases in MMP abundance, supporting an increase in proteolysis. A later phase demonstrated an increase in the relative abundance of TIMPs supporting decreased proteolysis. These temporal changes in MMP/TIMP relationships likely play a mechanistic role in the matrix-remodeling characteristic of TAAs.

\section{Early-phase Responses (24 Hours-1 Week)}

Results from this study have shown increases in MMP-8, MMP-12, and MMP-2 levels accompanied by a decrease in TIMP-1 levels during the early phase of aneurysm induction. These data imply a change in the ratio of MMPs to TIMPs, favoring a state of proteolysis, which is consistent with the initiation of aortic dilation and the beginning of aneurysm formation.
MMP-8 is highly expressed within circulating neutrophils and comprises the major neutrophil collagenase, showing 20fold specificity for fibrillar type I collagen compared with type III collagen or the nonfibrillar type IV collagen. ${ }^{18,19}$ Given the abundance of type I collagen in the aorta, MMP- 8 has been implicated in aneurysmal collagen degradation. ${ }^{20} \mathrm{MMP}-8$ has also been linked to aneurysmal disease as a marker for neutrophil infiltration into the aneurysmal wall. ${ }^{21}$ This report is the first to demonstrate the presence of active MMP- 8 in the developing TAA, as well as the first to demonstrate a unique temporal induction, showing an immediate increase in active MMP-8. Latent MMP-8 levels showed reciprocal changes consistent with release of preformed latent MMP-8 stores and conversion to the active form, with little additional de novo synthesis. In light of the fact that MMP-8 is primarily synthe- 
A.

\section{MMP-2}
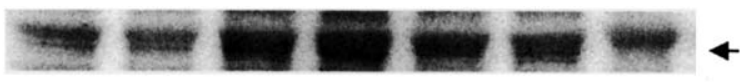

$62 \mathrm{kDa}$

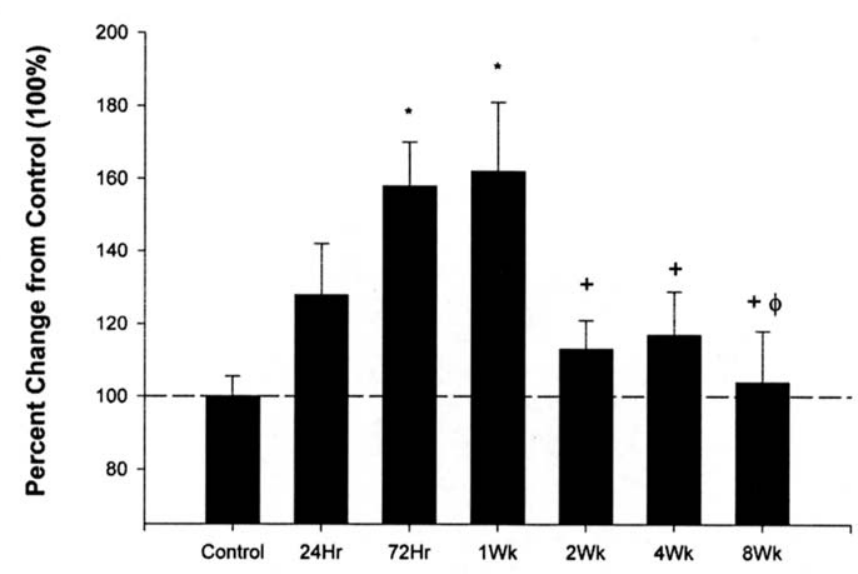

Time Following TAA Induction

\section{MMP-9}

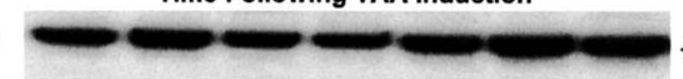

B.

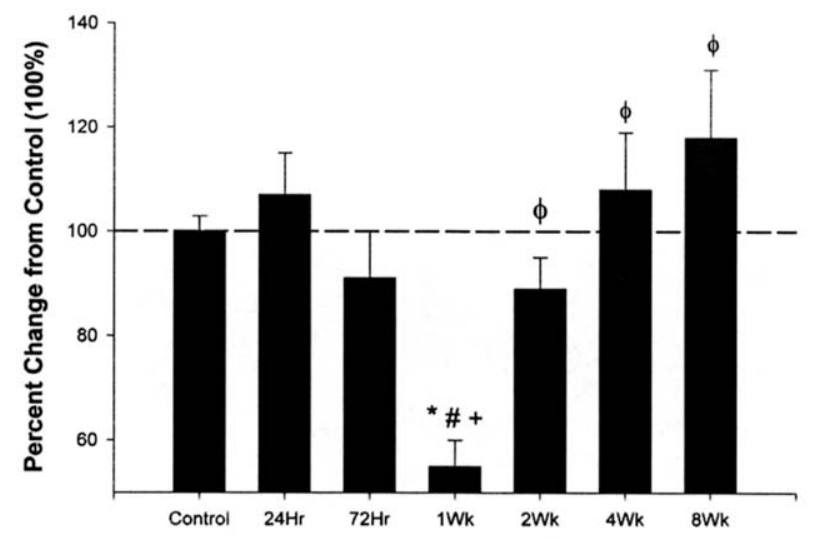

Time Following TAA Induction
Figure 3. A, The representative immunoblot shows the 62-kd band of active matrix metalloproteinase (MMP) 2. MMP-2 levels increased from control values at 72 hours and peaked in abundance at 1 week after the operation. MMP-2 levels then decreased to near control values at 2 weeks and at all time points afterward. ${ }^{*} P<.05$ from control, $+\boldsymbol{P}<.05$ from 72 hours, $\varphi P<.05$ from 1 week. $B$, The representative immunoblot shown in this figure shows the 88-kd band of active MMP-9. MMP-9 levels were decreased at 1 week; however, levels returned to control values at 2 weeks. MMP-9 levels were maximally increased at 8 weeks, although this increase was not significant from control values. ${ }^{*} \boldsymbol{P}<.05$ from control, $\# \boldsymbol{P}<.05$ from 24 hours, $+P<.05$ from 72 hours, $\phi P<.05$ from 1 week. TAA, Thoracic aortic aneurysm. sized and released by inflammatory cells, the crescendo of MMP-8 release observed early in this model of TAA is likely reflective of an inherent inflammatory response.

Levels of MMP-12, or macrophage elastase, have been found to be consistently increased in human aortic aneurysmal tissue when compared with that seen in control aortas. $^{22}$ Furthermore, it has been implicated as the major contributor to elastin degradation in aneurysmal tissue. ${ }^{9}$ In this study active MMP-12 levels were significantly increased over baseline values by 24 hours and then decreased to less than baseline values by 1 week. This early-phase response is consistent with macrophage infiltration induced by the surgical procedure. In addition, a parallel biphasic response in latent MMP-12 was observed, which is consistent with both preformed proenzyme release and de novo proenzyme synthesis.
MMP-2, predominantly expressed by smooth muscle cells and fibroblasts of mesenchymal origin, has long been implicated in the development of abdominal and thoracic aneurysms, both in human subjects and in experimental mouse models. ${ }^{6,23}$ Interestingly, in the current study active MMP-2 levels were found to be increased during the early phase of aneurysm development, peaking at 1 week after induction operations and then decreasing rapidly to baseline values at subsequent time points. Reciprocal changes were observed over time with latent MMP-2, which is consistent with early release of latent MMP-2 and rapid conversion to the active form. The activation of MMP-2 is a coordinated and localized cellular event requiring the interaction of several molecules and proteolytic steps. ${ }^{24}$ Thus changes in any of these steps or depletion of mesenchymal cell content might have led to attenuation of MMP-2 activational states. 


$$
\text { A. }
$$
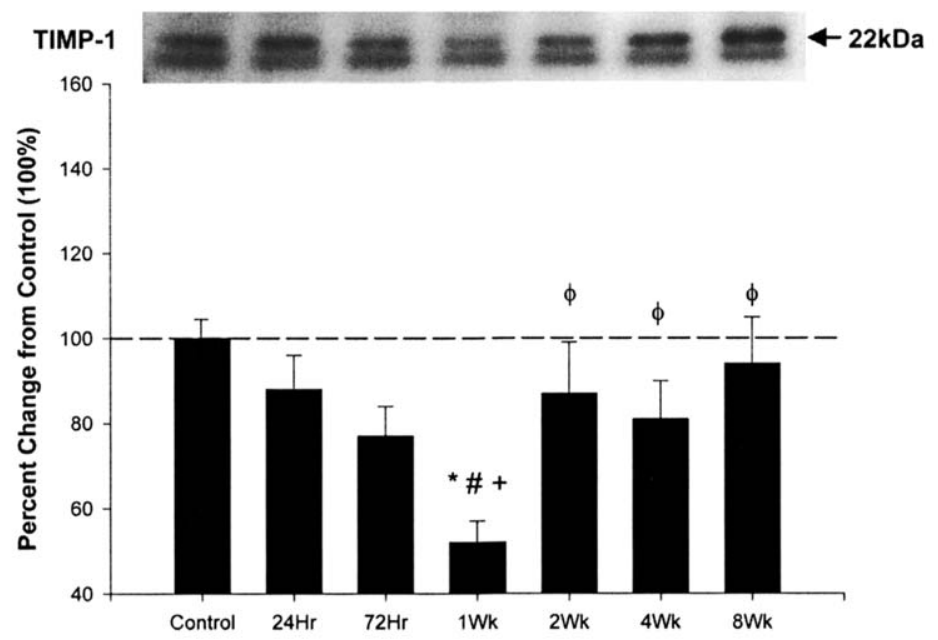

Time Following TAA Induction

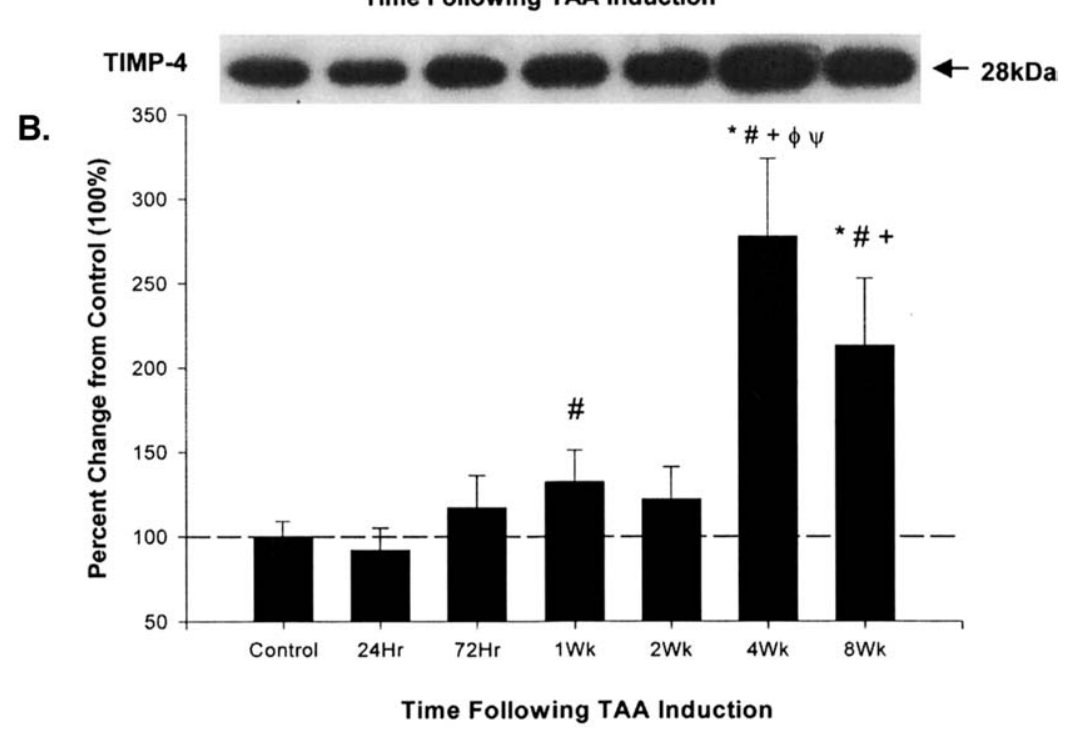

Figure 4. A, The representative immunoblot shows the 22-kd band of tissue inhibitor of metalloproteinases (TIMP) 1. TIMP-1 levels were found to be maximally decreased at 1 week before returning to control values at 2 weeks and beyond. ${ }^{*} \boldsymbol{P}<.05$ from control, \#P<.05 from 24 hours, $+P<.05$ from 72 hours, $\varphi P<.05$ from 1 week. B, The representative immunoblot shows the 28-kd band characteristic of TIMP-4. TIMP-4 levels were not changed from control values until 4 weeks, when levels were markedly increased from control values and remained increased at 8 weeks. ${ }^{*} P<.05$ from control, \#P< .05 from 24 hours, $+P<.05$ from 72 hours, $\phi P<$ .05 from 1 week, $\Psi P<.05$ from 2 weeks. TAA, Thoracic aortic aneurysm.
Late-phase Responses (2-8 Weeks)

The present study showed increases in active MMP-12 and active MMP-9 levels, as well as TIMP-4 levels, during the late phase of aneurysm formation. This was accompanied by continued aneurysm expansion through 4 weeks, followed by attenuation and plateau between 4 weeks and 8 weeks.

Active MMP-12 levels, which displayed an early response that was suppressed by 1 week, rebounded, reaching control levels by 2 weeks and peaked a second time at 4 weeks, before returning to baseline at 8 weeks. Because the early response was likely due to an acute inflammatory infiltrate, the second rebound response likely represents a shift from an acute inflammatory reaction to a more chronic reaction. Previous studies have shown chronic inflammation and elastin degradation in abdominal aneurysms. ${ }^{25}$ Moreover, elastin degradation products have been shown to have chemotactic properties for monocytes and macrophages, ${ }^{26}$ which might explain the rebound in active MMP-12 abundance observed during the late-phase response.

Several studies in human and experimental animal systems have demonstrated a role for MMP-9 in aneurysmal disease and have identified MMP-9 as the most abundant gelatinolytic MMP in aneurysm tissue. ${ }^{8,27}$ Infiltrating macrophages are the primary source of MMP-9 because mesenchymal cells do not normally produce MMP-9. Induction of experimental abdominal aortic aneurysms in MMP-9 ${ }^{-/-}$animals have demonstrated diminished elastin degradation and suppression of aneurysm formation. ${ }^{28,29}$ In this study active MMP-9 levels were decreased from reference control levels at 1 week and returned to control levels by 2 weeks, reaching the most increased values at 8 weeks after TAA induction. Although active MMP-9 levels were not increased until 
late in this study, latent MMP-9 levels were increased during the early time period ( 24 hours). These results might suggest that the role of MMP-9 in aneurysmal disease is in some measure related to alternative mechanisms other than through direct proteolysis.

The principle regulatory mechanism attenuating MMP activity in vivo is mediated through noncovalent interactions with a family of endogenous inhibitors or TIMPs. TIMP-1 and TIMP-4 are the predominant TIMP species expressed in the aortic vasculature. From previous studies, experimental aneurysm induction in TIMP- $1^{-1-}$ mice has shown increased aortic dilation. ${ }^{11,12}$ The present study is the first study to examine TIMP-4 in relation to aortic dilation and aneurysm formation. In this study a progressive loss of TIMP-1 was observed over the first week after surgical induction of TAA, followed by a return of TIMP-1 protein levels to baseline values. Interestingly, TIMP-4 levels were found to be markedly increased at 4 and 8 weeks after aneurysm induction operations. The 3-fold increase in TIMP-4 levels coincided with a relative decrease in the rate of aneurysmal expansion. This plateau in aortic dilation might represent a stoichiometric MMP/TIMP shift from an overall proteolytic state to a more homeostatic one.

\section{Limitations and Summary}

The goal of the current study was to assess MMP and TIMP abundance over the time course of aneurysm induction and formation in a periadventitial calcium chloride murine model of TAA. This study, however, is not without some limitations. First, the inflammatory nature of this model might limit its applicability to the TAAs in the ascending aorta, which in general tend to have less of an inflammatory component. Second, MMP abundance in this study was assessed by using antibodies directed against the latent and active forms of each MMP and comparing the determined levels at each time point with that of an unoperated control. Because of the limitations inherent in immunoblotting, comparisons between MMPs or TIMPs cannot be made directly. Third, the mouse aorta provides a small amount of tissue for biochemical analysis, and the immunoblotting techniques used in this experiment were therefore only directed at a small subset of the proteases and inhibitors known to play a role in vascular disease. Because neither TIMP-2, which plays a role in both the activation and inhibition of MMP-2, nor TIMP-3, which is known to interact with MMP-9, were analyzed, additional studies concerning these proteins might add additional insight. Lastly, as with any experimental animal model, this murine model of TAA is conducted in a separate species, and extrapolation to human TAAs must be done with caution.
Nevertheless, these findings show 2 phases of MMP abundance throughout the course of murine TAA formation. Unique MMP and TIMP temporal relationships were observed during TAA progression, which might hold both future diagnostic and therapeutic relevance.

\section{References}

1. Tang PC, Yakimov AO, Teesdale MA, et al. Transmural inflammation by interferon-gamma-producing $\mathrm{T}$ cells correlates with outward vascular remodeling and intimal expansion of ascending thoracic aortic aneurysms. Faseb J. 2005;19:1528-30.

2. Xiong W, Zhao Y, Prall A, et al. Key roles of CD4+ T cells and IFN-gamma in the development of abdominal aortic aneurysms in a murine model. J Immunol. 2004;172:2607-12.

3. Elefteriades JA. Natural history of thoracic aortic aneurysms: indications for surgery, and surgical versus nonsurgical risks. Ann Thorac Surg. 2002;74(suppl):S1877-80; discussion S1892-8.

4. Liapis CD, Paraskevas KI. The pivotal role of matrix metalloproteinases in the development of human abdominal aortic aneurysms. Vasc Med. 2003;8:267-71

5. Freestone T, Turner RJ, Coady A, et al. Inflammation and matrix metalloproteinases in the enlarging abdominal aortic aneurysm. Arterioscler Thromb Vasc Biol. 1995;15:1145-51.

6. Longo GM, Xiong W, Greiner TC, et al. Matrix metalloproteinases 2 and 9 work in concert to produce aortic aneurysms. J Clin Invest. 2002;110:625-32.

7. Longo GM, Buda SJ, Fiotta N, et al. MMP-12 has a role in abdominal aortic aneurysms in mice. Surgery. 2005;137:457-62.

8. Thompson RW, Holmes DR, Mertens RA, et al. Production and localization of 92-kilodalton gelatinase in abdominal aortic aneurysms. An elastolytic metalloproteinase expressed by aneurysm-infiltrating macrophages. J Clin Invest. 1995;96:318-26.

9. Curci JA, Liao S, Huffman MD, et al. Expression and localization of macrophage elastase (matrix metalloproteinase-12) in abdominal aortic aneurysms. J Clin Invest. 1998;102:1900-10.

10. Halloran BG, Baxter BT. Pathogenesis of aneurysms. Semin Vasc Surg. 1995;8:85-92.

11. Eskandari MK, Vijungco JD, Flores A, et al. Enhanced abdominal aortic aneurysm in TIMP-1-deficient mice. J Surg Res. 2005;123:289-93.

12. Ikonomidis JS, Gibson WC, Butler JE, et al. Effects of deletion of the tissue inhibitor of matrix metalloproteinases-1 gene on the progression of murine thoracic aortic aneurysms. Circulation. 2004;110(suppl):II268-73.

13. Schafers M, Riemann B, Kopka K, et al. Scintigraphic imaging of matrix metalloproteinase activity in the arterial wall in vivo. Circulation. 2004;109:2554-9.

14. Chiou AC, Chiu B, Pearce WH. Murine aortic aneurysm produced by periarterial application of calcium chloride. J Surg Res. 2001;99: 371-6.

15. Ikonomidis JS, Gibson WC, Gardner J, et al. A murine model of thoracic aortic aneurysms. J Surg Res. 2003;115:157-63.

16. Spinale FG, Coker ML, Thomas CV, et al. Time-dependent changes in matrix metalloproteinase activity and expression during the progression of congestive heart failure: relation to ventricular and myocyte function. Circ Res. 1998;82:482-95.

17. Thomas CV, Coker ML, Zellner JL, et al. Increased matrix metalloproteinase activity and selective upregulation in LV myocardium from patients with end-stage dilated cardiomyopathy. Circulation. 1998;97: 1708-15.

18. Chubinskaya S, Huch K, Mikecz K, et al. Chondrocyte matrix metalloproteinase-8: up-regulation of neutrophil collagenase by interleukin-1 beta in human cartilage from knee and ankle joints. Lab Invest. 1996;74:232-40.

19. Nagase H, Woessner JF Jr. Matrix metalloproteinases. J Biol Chem 1999;274:21491-4.

20. Wilson WR, Schwalbe EC, Jones JL, et al. Matrix metalloproteinase 8 (neutrophil collagenase) in the pathogenesis of abdominal aortic aneurysm. Br J Surg. 2005;92:828-33. 
21. Eliason JL, Hannawa KK, Ailawadi G, et al. Neutrophil depletion inhibits experimental abdominal aortic aneurysm formation. Circulation. 2005;112:232-40.

22. Annabi B, Shedid D, Ghosn P, et al. Differential regulation of matrix metalloproteinase activities in abdominal aortic aneurysms. $J$ Vasc Surg. 2002;35:539-46.

23. Thompson RW, Parks WC. Role of matrix metalloproteinases in abdominal aortic aneurysms. Ann N Y Acad Sci. 1996;800:15774.

24. Chakrabarti S, Patel KD. Matrix metalloproteinase-2 (MMP-2) and MMP-9 in pulmonary pathology. Exp Lung Res. 2005;31:599621.

25. Satta J, Laurila A, Paakko P, et al. Chronic inflammation and elastin degradation in abdominal aortic aneurysm disease: an immunohisto- chemical and electron microscopic study. Eur J Vasc Endovasc Surg. 1998;15:313-9.

26. Senior RM, Griffin GL, Mecham RP. Chemotactic activity of elastinderived peptides. J Clin Invest. 1980;66:859-62.

27. Koullias GJ, Ravichandran P, Korkolis DP, et al. Increased tissue microarray matrix metalloproteinase expression favors proteolysis in thoracic aortic aneurysms and dissections. Ann Thorac Surg. 2004;78: 2106-10.

28. Ikonomidis JS, Barbour JR, Amani Z, et al. Effects of deletion of the matrix metalloproteinase 9 gene on development of murine thoracic aortic aneurysms. Circulation. 2005;112(suppl):I242-8.

29. Pyo R, Lee JK, Shipley JM, et al. Targeted gene disruption of matrix metalloproteinase-9 (gelatinase B) suppresses development of experimental abdominal aortic aneurysms. J Clin Invest. 2000;105:1641-9.

\section{Interactive eLearning Activities http://learning.ctsnet.org}

The Joint Council on Thoracic Surgery Education was pleased to introduce a series of unique eLearning activities to CTSNet users at the AATS annual meeting in Philadelphia. Sponsored by several cardiothoracic surgical groups, this exciting new educational tool contains narrated videos of actual surgical procedures followed by a series of questions and an evaluation to earn Continuing Medical Education credit.

* Diagnosis and Management of Complications of Mitral Valve Repair

* Off Pump Coronary Artery Bypass

* OPCAB Debriefing

* Pediatric Cardiopulmonary Bypass Emergency Situations

\section{Experience these new educational tools today and provide us with your feedback. Go to: http://learning.ctsnet.org}

William E. Baumgartner, Chair

Joint Council on Thoracic Surgery Education

\section{Sponsored By:}

American Association for Thoracic Surgery

The Society of Thoracic Surgeons

European Association for Cardio-Thoracic Surgery

Children's Memorial Hospital 